\title{
Analysis of Clinical Efficacy of Glucose and Lipid Metabolism in Children with Growth Hormone Treatment of Turner Syndrome
}

\begin{abstract}
Bo Shi1
${ }^{1}$ Department of biology, Qingdao Universty, Shandong Qingdao, 266071, China

Abstract: Objective: To investigate the effects of growth hormone on Turner syndrome of glucose and lipid metabolism. Methods: 60 patients in August 2014 - February 2015 between patients with Turner syndrome treatment, all patients using recombinant human growth hormone (rhGH) treatment a year, to observe, record and compare the before and after treatment in patients with triglyceride (TG), total cholesterol (T-CHO), high density lipoprotein (HDL-C), atherogenic index (AI), fasting blood sugar (FBS), serum insulin (FINS), insulin resistance index (IRS), etc. level indicators. Results: After treatment of children AI decreased significantly, other TG, T-CHO, HDL-C, FBS, FINS and other glycolipid metabolism did not change significantly, P> 0.05. Conclusion Turner syndrome growth hormone treatment on lipid metabolism have a certain improvement, no significant effect on carbohydrate metabolism, worthy of clinical application.
\end{abstract}

Keywords: growth hormone; Turner syndrome; glucose and lipid metabolism

\section{Introduction}

Turner's syndrome, also known as congenital ovarian dysplasia syndrome, the $\mathrm{X}$ chromosome is caused by partial or complete deletion of chromosomal disorders [1]. The clinical manifestations of short stature, ear-bit low, breast wide apart, congenital ovarian hypoplasia. Due to congenital ovarian dysplasia, many children appear GH secretion [2]. As early as 1996, rhGH has been approved by the US FDA for the treatment of Turner syndrome [3]. However, some literature GH supplementation may have some effect on glucose and lipid metabolism in children. This test conducted a study to provide the clinical evidence for clinical practice. The following review of the research process.

\section{Materials and Methods}

\subsection{General Information}

60 patients in August 2014 - in patients with Turner syndrome between February 2015 treatment, including 42 males and 18 females, aged 1 year to 10 years, mean age $4.5 \pm 2.4$ years, weight $25.2 \pm 11.8 \mathrm{Kg}$, height 56.3 $\pm 20.1 \mathrm{~cm}$. Case inclusion criteria: (1) by the relevant diagnosed as Turner's syndrome; (2) the age of 10 years of age; (3) patients and their families informed consent; (4) the approval of the Ethics Committee. Case exclusion criteria: (1) with severe cognitive impairment; (2) in combination with other lipid metabolism diseases; (3) patients with severe heart, liver, kidney and other organ failure and other property; (4) do not meet the study By. 


\subsection{Methods}

All patients admitted to hospital after a routine examination and lipid metabolism testing, including TG, T-CHO, HDL-C, FBS, FINS and the like. IRS and other calculated values of $\mathrm{AI}$, where $\mathrm{AI}=$ (T-CHO-HDL-C) / HDL-C; IRS $=1 /$ (FBS * FINS). Children taking rhGH therapy, the dose may be individual differences depending on the condition, the treatment period of 1 year. These indicators were measured again after one year, compare the difference before and after treatment.

\subsection{Statistical Analysis}

Using SPSS19.0 statistical software to process data. Measurement data as mean \pm standard deviation () said. $\mathrm{P}<0.05$ indicate a statistically significant difference.

\section{Results}

Comparison, significantly lower after treatment in children with AI, other glycolipid metabolism did not change significantly, $\mathrm{P}>0.05$, Turner syndrome showed that growth hormone treatment for lipid metabolism have a certain improvement, no significant effect on carbohydrate metabolism, P>0.05. See Table 1 .

Table 1. Comparison of the inspection indicators before and after treatment $(\bar{x} \pm s$ )

\begin{tabular}{ccccccccc}
\hline Group & $\begin{array}{c}\text { Number } \\
\text { of cases } \\
(\text { examp } \\
\text { le })\end{array}$ & $\begin{array}{c}\text { TG } \\
(\mathrm{mmol} / \mathrm{L})\end{array}$ & $\begin{array}{c}\text { T-CHO } \\
(\mathrm{mmol} / \mathrm{L})\end{array}$ & $\begin{array}{c}\text { HDL-C } \\
(\mathrm{mmol} / \mathrm{L})\end{array}$ & AI & $\begin{array}{c}\text { FBS } \\
(\mathrm{mmol} / \mathrm{L})\end{array}$ & $\begin{array}{c}\text { FINS } \\
(\mathrm{mU} / \mathrm{L})\end{array}$ & IRS \\
\hline $\begin{array}{c}\text { Before } \\
\text { treatment } \\
\text { After }\end{array}$ & 60 & $1.14 \pm 0.79$ & $4.21 \pm 0.69$ & $1.52 \pm 0.39$ & $2.27 \pm 0.97$ & $9.3 \pm 2.9$ & $10.63 \pm 3.22$ & $3.87 \pm 2.32$ \\
treatment & 60 & $0.98 \pm 0.57$ & $3.92 \pm 0.52$ & $1.47 \pm 0.54$ & $1.76 \pm 0.89$ & $8.9 \pm 3.4$ & $11.02 \pm 3.57$ & $4.03 \pm 1.96$ \\
$\mathrm{t}$ & - & 1.586 & 0.402 & 0.283 & 5.283 & 0.454 & 0.238 & 0.397 \\
$\mathrm{P}$ & - & $>0.05$ & $>0.05$ & $>0.05$ & $<0.01$ & $>0.05$ & $>0.05$ & $>0.05$ \\
\hline
\end{tabular}

\section{Discussion}

rhGH is a synthetic growth hormone, growth hormone produced by the body as self, have to adjust the endocrine system, activate and maintain the normal operation of the immune system and so on. rhGH can induce liver cells, muscle cells.

The GH media, regulating blood lipids play an indirect role, but also can produce physiological effects by acting on target cells and thus have a direct effect.

The results of this study showed that in children after treatment was significantly lower AI, other glycolipid metabolism did not change significantly, P> 0.05 , Turner syndrome showed that growth hormone therapy for improving lipid metabolism have a certain effect on carbohydrate metabolism no influences. Studies have shown, Turner syndrome, the presence of lipid accumulation in vivo situation of children after treatment TG, T-CHO, HDL-C decreased, but the difference was not significant, but the atherogenic index decreased significantly, indicating that lipid rhGH some improvement in metabolism, can reduce atherosclerosis lipid accumulation caused by atherosclerosis. Overall, a good syndrome rhGH treatment Turner, while improving lipid metabolism had no significant effect on glucose metabolism, safe and reliable.

In summary, the growth hormone treatment Turner syndrome on lipid metabolism have a certain improvement, no significant effect on carbohydrate 
metabolism, worthy of clinical application.

\section{References:}

1. Wangrui Fang, Dong Zhi-ya, Wang Wei and other growth hormone therapy Turner syndrome into effect the year-end height and its influencing factors [J] Journal of Endocrinology and Metabolism, 2011,27 (11): 901-905.

2. Guo Song, Du Min Alliance, Chen Hongshan and other studies in patients with metabolic syndrome prevalence and associated risk factors $[\mathrm{J}]$ single center in Guangzhou Turner syndrome Chinese Journal of Endocrinology and Metabolism, 2014,30 (12): $1074-1079$.

3. Clinical and cytogenetic studies of patients [J] Li Chuan, Fan Xin, Luo Jingsi etc. .148 Example Turner syndrome China Maternal and Child Health, 2014,29 (22): 3630-3633. 